\title{
Degree of Ionization in MALDI of Peptides: Thermal Explanation for the Gas-Phase Ion Formation
}

\author{
Yong Jin Bae, ${ }^{1}$ Young Sik Shin, ${ }^{1}$ Jeong Hee Moon, ${ }^{2}$ Myung Soo Kim ${ }^{1}$ \\ ${ }^{1}$ Department of Chemistry, Seoul National University, Seoul 151-742, Korea \\ ${ }^{2}$ Medical Proteomics Research Center, KRIBB, Daejeon 305-806, Korea
}

\begin{abstract}
Degree of ionization (DI) in matrix-assisted laser desorption ionization (MALDI) was measured for five peptides using $\alpha$-cyano-4-hydroxycinnanmic acid (CHCA) as the matrix. Dls were low $10^{-4}$ for peptides and $10^{-7}$ for CHCA. Total number of ions (i.e., peptide plus matrix) was the same regardless of peptides and their concentration, setting the number of gas-phase ions generated from a pure matrix as the upper limit to that of peptide ions. Positively charged cluster ions were too weak to support the ion formation via such ions. The total number of gas-phase ions generated by MALDI, and that from pure $\mathrm{CHCA}$, was unaffected by the laser pulse energy, invalidating laser-induced ionization of matrix molecules as the mechanism for the primary ion formation. Instead, the excitation of matrix by laser is simply a way of supplying thermal energy to the sample. Accepting strong Coulomb attraction felt by cations in a solid sample, we propose three hypotheses for gas-phase peptide ion formation. In Hypothesis 1 , they originate from the dielectrically screened peptide ions in the sample. In Hypothesis 2, the preformed peptide ions are released as part of neutral ion pairs, which generate gas-phase peptide ions via reaction with matrix-derived cations. In Hypothesis 3 , neutral peptides released by ablation get protonated via reaction with matrix-derived cations.
\end{abstract}

Key words: Degree of ionization, Peptide, MALDI, Ion formation mechanism, Laser fluence dependence, Preformed ion, Gas phase proton transfer

\section{Introduction}

$\mathrm{M}$ atrix-assisted laser desorption ionization (MALDI) [1-3] is widely used in mass spectrometry for biological molecules. Inspired by its spectacular performance, especially for peptides and proteins, there were many attempts to explain the ion formation process involved, most of which turned out to be inappropriate upon further tests. It is a bit of a surprise to note that there is no general consensus on how peptide ions form in MALDI even after more than 20 years of its invention. The

Electronic supplementary material The online version of this article (doi:10.1007/s13361-012-0406-y) contains supplementary material, which is available to authorized users.

Correspondence to: Myung S. Kim; e-mail: myungsoo@snu.ac.kr following two hypotheses seem to prevail at the moment and, hence, are worth further tests. One is the gas-phase proton transfer (GPPT) hypothesis [4-8] proposed by Knochenmuss et al. and the other is the lucky survivor (LS) hypothesis [9-11] proposed by Karas et al. In the GPPT hypothesis, pooling of excitons in photoexcited matrix molecules results in the formation of matrix-derived primary ions. An analyte (A, a peptide in this work) is desorbed as a neutral but gets protonated by the transfer of a proton from a matrix-derived ion such as $\mathrm{MH}^{+}$(e.g., $\mathrm{MH}^{+}+\mathrm{A} \rightarrow \mathrm{M}+\mathrm{AH}^{+}$). The main assumptions in the LS hypothesis are as follows. A peptide is present as a protonated form, or a preformed ion form, inside a solid sample and desorbs in the form of $\mathrm{AH}^{+}$or as clusters consisting of $\mathrm{AH}^{+}$, matrix neutrals, and counter anions. In addition to the intact $\mathrm{AH}^{+}$ ions, positively charged clusters contribute to the peptide ion $\left(\mathrm{AH}^{+}\right)$signal through neutral loss after proton-transfer neutral- 
ization. A singly charged peptide ion surviving this process is called the lucky survivor. Two main differences between these hypotheses are to be emphasized. First, a peptide desorbs in its neutral form in the GPPT hypothesis whereas in a charged form in the LS hypothesis. Second, the electronic excitation of matrix molecules contributes to primary ion formation in the GPPT hypothesis whereas a matrix is simply a medium converting laser energy into thermal energy in the LS hypothesis.

Even though the degree of ionization (DI, number of ions formed $\div$ number of molecules in a sample) in MALDI is a quantity that is related to the ion formation mechanism, there were no serious attempts to utilize DI data for such an investigation, probably because no accurate data were available $[1,12-15]$. DIs cannot be readily estimated from MALDI spectra due to various experimental uncertainties such as sample homogeneity, laser spot size, ion fragmentation, and transmission, etc. Recently, we developed a rather reliable method to determine DI and collected the data for some compounds [16].

Even when DI data for peptides are available, interpreting them can be troublesome because the protonation occurring at some stage must be accommodated. The mechanism for the gas-phase ion formation in MALDI of salts may be simpler because their cations and anions will be present as preformed ions in solid samples and, hence, MALDI of salts might simply be a process of releasing preformed ions into the gas phase. In a previous work [16], we studied the degree of ionization in MALDI of three salts, benzyltriphenylphosphonium chloride (BTPP-Cl), 1-butyl-3-methylimidazolium hexafluorophosphate $\left(\mathrm{BMI}_{-} \mathrm{PF}_{6}\right)$, and trihexyltetradecylphosphonium bis(2,4,4-trimethylpentyl)phosphonate (THTDP-BTMPP). Since the results for the three salts were virtually the same, we will pick BTPP-Cl as the prototype of salts and compare its data with those of peptides. DI for the positive ions from BTPP-Cl was very small, $<10^{-3}$, and that of the matrix $\alpha$-cyano-4-hydroxycinnanmic acid (CHCA) was even smaller, around $10^{-7}$. The matrix and analyte suppression effects $[7,8,16]$ were also observed. To explain the experimental results, the release of a preformed ion in a solid sample into the gas phase was assumed to be very difficult due to the strong Coulomb attraction by a counter ion(s). Then, two hypotheses were proposed to explain the formation of gas-phase $\mathrm{BTPP}^{+}$, even though with small DI. In Hypothesis 1, we proposed that a tiny fraction of the preformed $\mathrm{BTPP}^{+}$ions in the solid were dielectrically screened from counter anions by matrix neutrals and hence were easily released into the gas phase. In Hypothesis 2, we proposed that the preformed $\mathrm{BTPP}^{+}$ions in the solid were released as part of neutral clusters or ion pairs, then lost counter anions via reactions with matrix-derived cations, as illustrated below.

$$
\mathrm{BTPP}^{+} \mathrm{X}^{-}+\mathrm{MH}^{+} \rightarrow \mathrm{BTPP}^{+}+\mathrm{M}+\mathrm{HX}
$$

Reaction 1 suggests that the proton transfer may participate, even though indirectly, in the formation of the gas-phase $\mathrm{BTPP}^{+}$.
In this work, we have extended our study to peptides. Because peptides and salts are chemically very different (i.e., covalent versus ionic compounds), we expected to observe significant difference between the two groups of compounds in the magnitude of DI and the concentration dependence (suppression effect). Against our expectation, however, the overall features for these two groups turned out to be similar. Implications of the experimental results on the ion formation mechanism in MALDI will be presented.

\section{Experimental}

Details of the homebuilt MALDI-TOF instrument and its operation were reported previously $[17,18]$. The instrument consists of an ion source with delayed extraction, a linear TOF analyzer, an ion gate, a second-stage analyzer equipped with a reflectron with linear-plus-quadratic (LPQ) potential inside, and a microchannel plate detector (MCP, \#31849“ Photonis USA, Sturbridge, MA, USA). A deflection system [19] is installed in front of the ion gate to eliminate postsource decay (PSD) products formed in the linear TOF analyzer. With 13 grids on the ion path, the theoretical transmission was 0.283 . The $337 \mathrm{~nm}$ output from a nitrogen laser (MNL100, Lasertechnik Berlin, Berlin, Germany) focused by an $\mathrm{f}=100 \mathrm{~mm}$ lens was used. In addition to the prompt ions and their in-source decay (ISD) products, PSD products of the prompt ions and ISD products appear in a MALDI-TOF spectrum recorded by the present apparatus [20]. That is, the total intensities of the analyte- and matrixderived ions can be measured from a mass spectrum.

\section{Sample Preparation}

$\mathrm{Y}_{5} \mathrm{X}(\mathrm{X}=\mathrm{E}, \mathrm{K}$, and $\mathrm{R})$ were purchased from Peptron (Daejeon, Korea). $\mathrm{Y}_{6}$, Angiotensin II (DRVYIHPF), and CHCA were purchased from Sigma (St. Louis, MO, USA). Aqueous solution of a peptide was mixed with 1:1 water/ acetonitrile solution of CHCA. $1 \mu \mathrm{l}$ of a mixture was loaded and vacuum-dried. The amounts of CHCA and a peptide loaded were $25 \mathrm{nmol}$ and 0-30 pmol, respectively.

\section{Method}

A brief summary of the method to measure DI is as follows [16].

\section{Sample Homogeneity}

Peptide-CHCA mixtures were vacuum-dried to prepare more homogeneous samples than by air-drying (Supplementary Material).

\section{Laser Pulse Energy}

The threshold pulse energy for 1 pmol of each peptide in $25 \mathrm{nmol} \mathrm{CHCA}$ was $0.75 \mu \mathrm{J} /$ pulse [16], which decreased a 
little with the analyte concentration. The $0.75 \mu \mathrm{J} /$ pulse will be called the threshold. The 2-10 times the threshold energy was used in the study.

\section{Spectral Collection over an Extended Region}

The total number of ions emitted from a sample (with the area $A_{S}$ ) was estimated from those emitted from a representative spot (with the area $A_{R}$ ) by multiplying the area ratio, $A_{S} / A_{R}$. $A_{R}$ much larger than a laser spot size was used because the measurement of ion emission from a focused laser spot would involve some uncertainties. First, it is difficult to define and accurately determine $A_{R}$ in such measurements. Second, the effective magnitude of $A_{R}$ may increase with the laser fluence as pointed out by Beavis [21]. Third, some materials outside the spot may melt and flow into the spot making the measurement of the spot size even more difficult.

In each experiment, we fixed the laser fluence (pulse energy $\div$ irradiated area) by fixing the pulse energy and the focusing optics, kept irradiating a spot, and displayed the intensities of some prominent analyte- and matrix-derived peaks summed over 20 shots. Irradiation stopped when hardly any ions appeared, which took $500-1500$ shots. Then, we moved the sample stage along one direction (positive $\mathrm{x}$ ) by a length smaller than the laser spot length, collected data, moved the stage again, etc. until a desired length along the $x$ axis was covered. Then, after moving the stage along y by a small length and collecting data, we moved it along the negative $\mathrm{x}$ direction, eventually collecting ions from an extended region of parallelogram with a dimension of $85 \mu \mathrm{m} \times 250 \mu \mathrm{m}$. The dimension itself was measured from the burn mark on the sample formed by the laser with a CCD camera (AM413T; Dinolite, Naarden, The Netherlands). Experimental evidence for rather complete depletion of the samples is presented in Supplementary Material.

\section{MCP Gain Calibration and Saturation Check}

The method to calibrate the gain of the MCP detector was reported previously [22]. Tests were made to ensure that the detector saturation $[23,24]$ did not occur (to be explained).

\section{Correction for the Ion Loss by Fragmentation}

Since the in-source decay (ISD) of a prompt ion occurs mostly within the delay time [25], the ion loss due to rapid dissociation can be corrected simply by including the ISD intensities in DI measurement. Correcting for the ion loss due to post-source dissociation is more troublesome because the PSD spectrum records product ions formed only between the end of the deflection system and the reflectron entrance (PSD region). In terms of flight time, PSD region is roughly located half way between the ion source and the detector. Since the flight time through this region was oneninth of the total flight time, we assumed that the total post-source dissociation product intensity would be roughly nine times the intensity measured by PSD [16]. For PSD products with $\mathrm{m} / \mathrm{z}$ larger than $90 \%$ of the precursor ion, the correction factor of 2 was used because such ions passed the deflection system. Our estimation based on the first order kinetics suggested $\pm 20 \%$ error in this step and $\pm 10 \%$ error in DI. The latter was smaller than the random errors in DI measurement.

\section{Determination of DI}

All of the MALDI spectra collected over an extended region were summed and corrected for the MCP gain and the fragmentation loss. Also made was the ion transmission correction $(28.3 \%$ transmission through the instrument and $62 \%$ entrance to microchannels in MCP). The number of each analyte-derived ion was added to get the sum. Then, the area ratio $\left(A_{S} / A_{R}\right)$ was multiplied to the sum to estimate the total number of the analyte-derived ions generated from the whole sample. Finally, this total number of ions was divided by the number of the analyte molecules loaded on the target to get DI. A similar method was used to determine DI for the matrix.

\section{Results and Discussion}

\section{Spectra}

The MALDI spectra for $\mathrm{Y}_{5} \mathrm{R}$, DRVYIHPF, and $\mathrm{Y}_{5} \mathrm{E}$ obtained with 10 pmol each in $25 \mathrm{nmol}$ CHCA are shown in Figure 1. Six times the threshold laser pulse energy (i.e., $4.5 \mu \mathrm{J} /$ pulse) was used. The MALDI spectra for $\mathrm{Y}_{5} \mathrm{~K}$ and $\mathrm{Y}_{6}$ obtained under the same condition are in Supplementary Material. The most prominent CHCA-derived peaks were $[\mathrm{nCHCA}+\mathrm{H}]^{+},\left[\mathrm{nCHCA}+\mathrm{H}-\mathrm{H}_{2} \mathrm{O}\right]^{+}$, and $[\mathrm{nCHCA}+\mathrm{H}-$ $\left.\mathrm{CO}_{2}\right]^{+}$with $\mathrm{n}=1$ and 2 . These matrix-derived acidic cations will be collectively called $\mathrm{M}^{\prime} \mathrm{H}^{+}$to differentiate them from $\mathrm{MH}^{+}$for $[\mathrm{CHCA}+\mathrm{H}]^{+}$. $\left[\mathrm{nCHCA}+\mathrm{H}-\mathrm{H}_{2} \mathrm{O}\right]^{+}$and $[\mathrm{nCHCA}$ $\left.+\mathrm{H}-\mathrm{CO}_{2}\right]^{+}$must be the ISD products of $[\mathrm{nCHCA}+\mathrm{H}]^{+}$ because they were the main peaks in the PSD spectra of $[\mathrm{nCHCA}+\mathrm{H}]^{+}$(in Supplementary Material). In the PSD spectrum of $\left[\mathrm{Y}_{5} \mathrm{R}+\mathrm{H}\right]^{+}$(in Supplementary Material), b, y, z, immonium $\mathrm{Y}$, and $\left[\mathrm{Y}_{5} \mathrm{R}+\mathrm{H}-\mathrm{NH}_{3}\right]^{+}$ions appeared. These ions were formed by ISD also and appeared in the MALDI spectrum together with some neutral loss peaks such as $\left[\mathrm{Y}_{5} \mathrm{R}+\mathrm{H}-\mathrm{CO}_{2}\right]^{+}$. Even though alkali metal adduct peaks also appeared, they were not marked because they were much weaker than CHCA-derived peaks. They were included in measuring the number of $\mathrm{Y}_{5} \mathrm{R}$-derived ions, even though their contribution was insignificant. For angiotensin II, a, b, b- $\mathrm{NH}_{3}, \mathrm{y}$, immonium ions, and $\left[\mathrm{DRVYIHPF}+\mathrm{H}-\mathrm{NH}_{3}\right]^{+}$ions generated by ISD and PSD appeared in the MALDI spectrum. Neutral loss and adduct ions also appeared, as for $\mathrm{Y}_{5} \mathrm{R}$. For $\mathrm{Y}_{5} \mathrm{E}, \mathrm{a}, \mathrm{b}, \mathrm{y}$, and immonium, $\mathrm{Y}$ were the main fragment ions. 


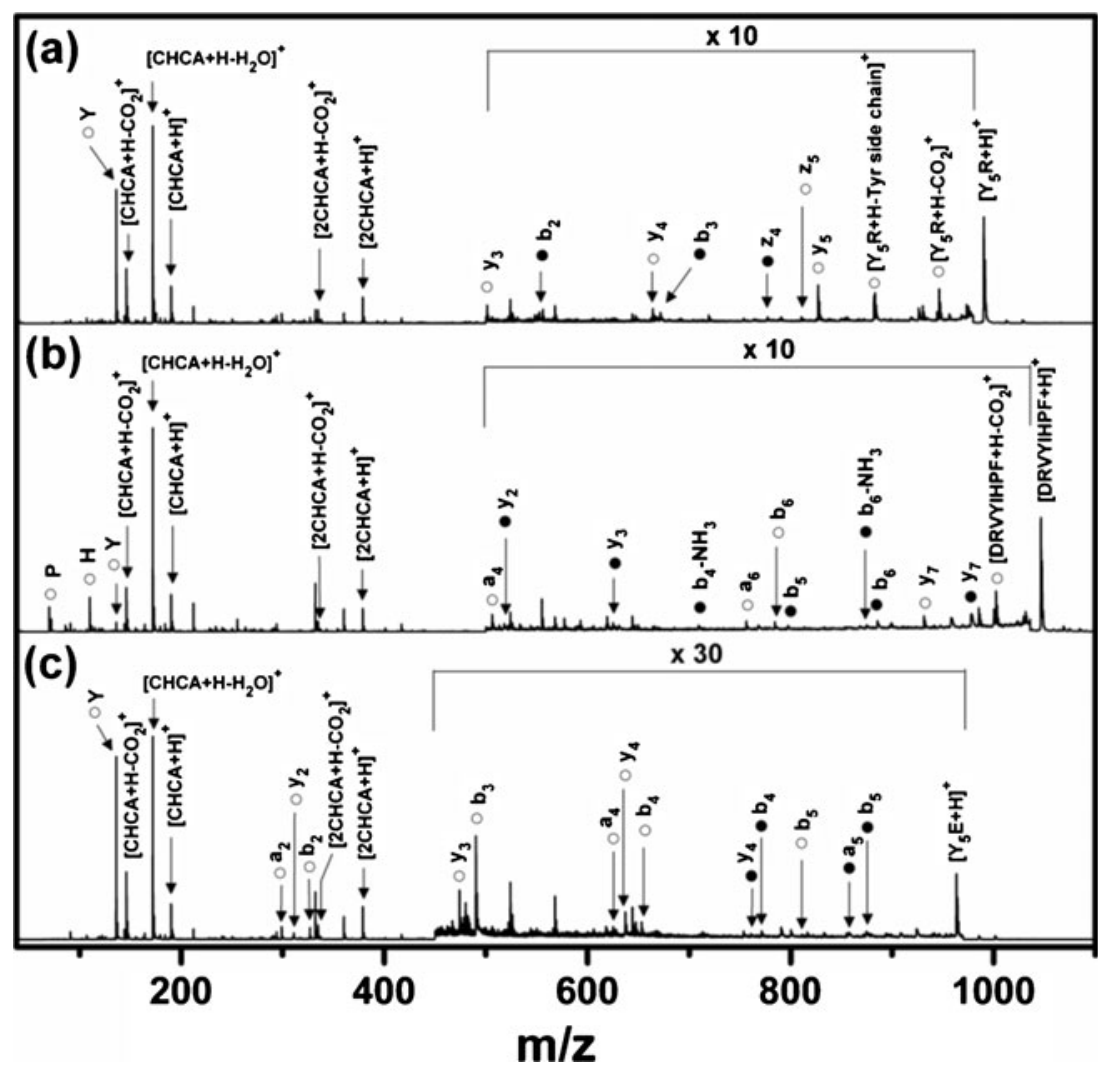

Figure 1. The MALDI spectra for (a) $Y_{5} R$, (b) DRVYIHPF, and (c) $Y_{5} E$ obtained with 10 pmol each in 25 nmol CHCA. Six times the threshold laser pulse energy (i.e., $4.5 \mu \mathrm{J} /$ pulse) was used. Some matrix- and analyte-derived ions are marked. Open and filled circles represent ISD and PSD products, respectively, of the analytes

\section{Search for Cluster Ions}

In the LS hypothesis of Karas, a preformed ion can be emitted directly from the sample or as part of cluster ions consisting of the preformed ions, matrix neutrals, and counter anions. In our previous study of BTPP-Cl [16], we could not find positive cluster ions containing $\mathrm{BTPP}^{+}$. This suggested either that the gas-phase $\mathrm{BTPP}^{+}$was not formed via cluster ions or that the lifetimes of the cluster ions were shorter than the mass spectrometric time scale. Even in the second case, the ion formation mechanism can be treated as if the gas-phase $\mathrm{BTPP}^{+}$is formed directly from the preformed one in the solid sample.

In MALDI of CHCA, [CHCA - $\mathrm{H}^{-}$and its products almost completely dominate the negative ion spectrum [26, 27]. Following Karas [11], let us take the positive cluster ions such as $\left[\mathrm{Y}_{5} \mathrm{R}+\mathrm{H}\right]_{\mathrm{n}}{ }^{\mathrm{n}+}[\mathrm{CHCA}]_{\mathrm{x}}[\mathrm{CHCA}-\mathrm{H}]_{\mathrm{m}}{ }^{\mathrm{m}-}$ or $\left[\mathrm{Y}_{5} \mathrm{R}\right.$ $+\mathrm{nH}]^{\mathrm{n}+}[\mathrm{CHCA}]_{\mathrm{x}}[\mathrm{CHCA}-\mathrm{H}]_{\mathrm{m}}{ }^{\mathrm{m}^{-}}$with $\mathrm{n}>\mathrm{m}$ as the intermediates for the formation of $\left[\mathrm{Y}_{5} \mathrm{R}+\mathrm{H}\right]^{+}$. We searched for the above ions, which might appear as ISD or PSD peaks in a MALDI spectrum. Search was made for cluster ions with $\mathrm{n}$ up to 3 and with $x$ up to 2. In the case of CHCA-MALDI spectrum of $Y_{5} R$, the only cluster ion found was $\left[Y_{5} R+H+\right.$ $\mathrm{CHCA}]^{+}$, whose intensity was $1 \%$ of the total. In addition, its ISD peaks $\left[\mathrm{Y}_{5} \mathrm{R}+\mathrm{H}+\mathrm{CHCA}-\mathrm{CO}_{2}\right]^{+}$and $\left[\mathrm{Y}_{5} \mathrm{R}+\mathrm{H}+\right.$ $\left.\mathrm{CHCA}-\mathrm{H}_{2} \mathrm{O}\right]^{+}$appeared weakly. The same was the case for
DRVYIHPF, while cluster ions were absent for $\mathrm{Y}_{5} \mathrm{E}$. Hence, we concluded that the peptide ion formation via cluster ions could be ignored, as also found for salts [16].

\section{Degree of Ionization (DI)}

CHCA-MALDI experiments were carried out for samples containing $25 \mathrm{nmol}$ of CHCA and $0-30$ pmol of peptides. Here we show the results obtained with the laser pulse energy of $4.5 \mu \mathrm{J} / \mathrm{pulse}$, or $6 \times$ threshold. Three or more replicate measurements for three or more samples were made at each concentration. Random errors of one standard deviation are quoted. The numbers of $\mathrm{Y}_{5} \mathrm{R}-$ and CHCAderived ions, their sum, and DIs from $\mathrm{Y}_{5} \mathrm{R}$-in-CHCA experiments are listed in Table 1. Similar data from angiotensin II-, $\mathrm{Y}_{5} \mathrm{E}-, \mathrm{Y}_{5} \mathrm{~K}$-, and $\mathrm{Y}_{6}$-in-CHCA experiments are in Supplementary Material.

Even though DI for each peptide and that for CHCA changed with the peptide concentration in the solid sample, the total number of gas-phase ions (i.e., the sum of the peptide- and matrix-derived ions) remained constant. In particular, it was the same as the number of ions generated from pure CHCA, setting it as the upper limit to the number of peptide-derived ions that could be generated by MALDI. Since the concentration dependences are similar for the five peptides, let us compare the DI data obtained for $1 \mathrm{pmol}$ 
Table 1. The Numbers of $Y_{5} R$ - and CHCA-Derived Positive Ions, Their Sum, and DIs of $Y_{5} R$ and $C H C A$ in CHCA-MALDI Versus the Amount of ${ }_{5} R$ in $25 \mathrm{nmol} \mathrm{CHCA}$

\begin{tabular}{|c|c|c|c|c|c|}
\hline \multirow[b]{2}{*}{$\mathrm{Y}_{5} \mathrm{R}, \mathrm{pmol}$} & \multicolumn{3}{|c|}{ Number of ions from sample } & \multicolumn{2}{|c|}{ DI } \\
\hline & $\mathrm{Y}_{5} \mathrm{R}, 10^{8}$ & CHCA, $10^{9}$ & Sum, $10^{9}$ & $\mathrm{Y}_{5} \mathrm{R}, 10^{-4}$ & CHCA, $10^{-7}$ \\
\hline 0 & - & $1.8 \pm 0.2$ & $1.8 \pm 0.2$ & - & $1.2 \pm 0.1$ \\
\hline 0.1 & $0.16 \pm 0.03$ & $1.7 \pm 0.6$ & $1.7 \pm 0.6$ & $2.6 \pm 0.5$ & $1.1 \pm 0.4$ \\
\hline 0.3 & $0.51 \pm 0.15$ & $1.7 \pm 0.1$ & $1.7 \pm 0.1$ & $2.8 \pm 0.9$ & $1.2 \pm 0.1$ \\
\hline 1 & $1.4 \pm 0.4$ & $1.5 \pm 0.2$ & $1.6 \pm 0.3$ & $2.3 \pm 0.6$ & $0.95 \pm 0.13$ \\
\hline 3 & $3.8 \pm 0.4$ & $1.4 \pm 0.3$ & $1.7 \pm 0.3$ & $2.1 \pm 0.2$ & $0.91 \pm 0.20$ \\
\hline 10 & $8.5 \pm 1.8$ & $0.71 \pm 0.20$ & $1.6 \pm 0.4$ & $1.4 \pm 0.3$ & $0.48 \pm 0.13$ \\
\hline 30 & $13 \pm 3$ & $0.28 \pm 0.11$ & $1.5 \pm 0.4$ & $0.70 \pm 0.17$ & $0.19 \pm 0.07$ \\
\hline
\end{tabular}

analyte in $25 \mathrm{nmol}$ matrix. They were $(2.3 \pm 0.6) \times 10^{-4}$ for $\mathrm{Y}_{5} \mathrm{R},(2.5 \pm 0.5) \times 10^{-4}$ for angiotensin II, $(2.2 \pm 0.1) \times 10^{-4}$ for $\mathrm{Y}_{5} \mathrm{~K},(1.8 \pm 0.2) \times 10^{-4}$ for $\mathrm{Y}_{6}$, and $(1.6 \pm 0.1) \times 10^{-4}$ for $\mathrm{Y}_{5} \mathrm{E}$. Even though DI tends to increase a little as a peptide gets more basic, the changes look too small to draw any conclusion. More surprising is that they are on the same order of magnitude as $(7.5 \pm 1.4) \times 10^{-4}$ for BTPP-Cl [16]. Present results are in good agreement with $3 \times 10^{-4}$ for insulin in sinapinic acid [12] However, it is smaller than $10^{-3}-10^{-1}$ speculated by Hillenkamp and Karas [1].

For samples with low peptide concentration, DIs for CHCA were virtually the same regardless of the analyte (i.e., around $10^{-7}$ for the samples containing $\mathrm{Y}_{5} \mathrm{X}(\mathrm{X}=\mathrm{E}, \mathrm{K}$, $\mathrm{R}$, and $\mathrm{Y}$ ), angiotensin II, and BTPP-Cl). DI for CHCA is rather close to $10^{-7}-10^{-6}$ estimated from the data reported by Sundqvist et al. [15], but much smaller than $10^{-5}$ reported by Puretzky and Geohegan [13], and even smaller than $10^{-5}-10^{-3}$ speculated by Hillenkamp and Karas [1].

The errors quoted for the experimental DI data are random ones calculated from replicate measurements. Various error sources such as the sample inhomogeneity and the fluctuation of the detector gain would be responsible for the random errors. For repeated measurements (such as reported in the second item of Supplementary Material), random errors were found to be around $\pm 20 \%$ or less. The main source of uncertainty for the present results is the ion transmission efficiency. We attempted to estimate the magnitude of this systematic error by installing apertures at several locations inside the instrument and by changing the instrument tuning. We guess that the magnitude of the systematic error would be comparable to that of the random one, certainly smaller than $\pm 50 \%$.

\section{Laser Pulse Energy Dependence of Gas-Phase Ion Formation}

For salts, the total number of ions emitted from a sample was similar regardless of the laser pulse energy in the range (4-10) $\times$ threshold. Measurement at lower energy was difficult because the burn mark for the parallelogram did not form cleanly. In this work, we obtained similar results for the peptides. DIs of $\mathrm{Y}_{5} \mathrm{R}$ and $\mathrm{CHCA}$-derived ions and the total number of ions from a sample containing 1 pmol $\mathrm{Y}_{5} \mathrm{R}$ in $25 \mathrm{nmol}$ CHCA versus laser pulse energy, (4-10) $\times$ threshold, are shown in Figure 2. Even though we could not measure DIs below $4 \times$ threshold, we do not expect drastic changes in the ion formation mechanism at lower energy because the mass spectral patterns did not change much (see Supplementary Material). The trend observed for the
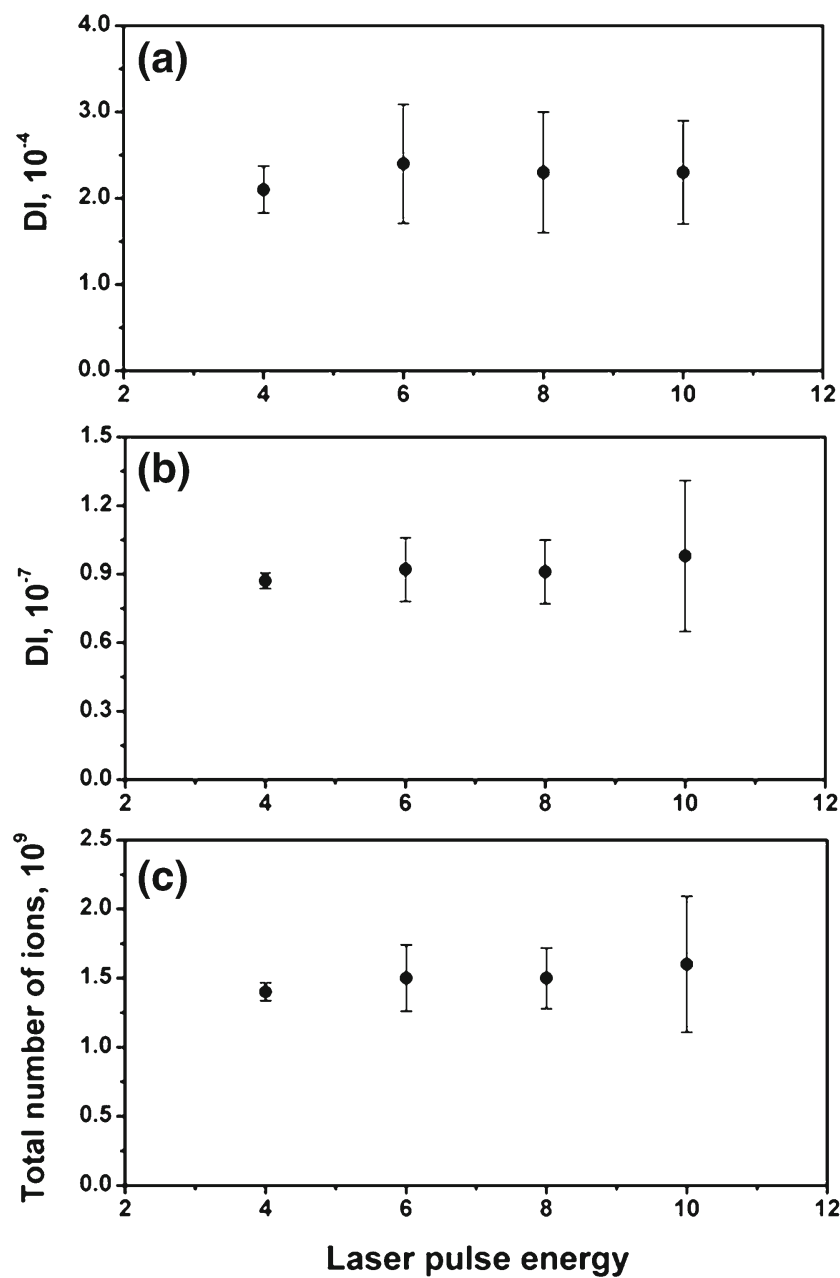

Figure 2. Dls of (a) $Y_{5} R$, (b) $\mathrm{CHCA}$, and (c) total number of ions (sum of $Y_{5} R$ - and CHCA-derived ions) in MALDI of 1 pmol $Y_{5} R$ in $25 \mathrm{nmol} C H C A$ versus the laser pulse energy. The threshold energy (i.e., $0.75 \mu \mathrm{J} /$ pulse) is used as the energy unit 
other peptides was similar. Recently, Musapelo and Murray [28] reported the formation of nanoparticles in ambient MALDI plumes. We do not know whether such particles are formed in vacuum MALDI also and, if they are, how they will affect the fluence dependence of DI.

Our finding that DI of a peptide is unaffected by laser pulse energy may look totally absurd because it is common knowledge in MALDI that an ion signal in a single shot spectrum increases rapidly with the laser fluence near the threshold. For example, when the fluence at the threshold doubles, ion intensity increases by a factor of a few hundred. Such a relation is often expressed as $Y \propto H^{\mathrm{m}}$ (with $\mathrm{m}=5-10$ ) where $Y$ and $H$ are the ion intensity and the laser fluence, respectively [3]. A closer look at $Y$ versus $H$ data in the literature $[3,29,30]$ shows that the ion intensity $(Y)$ saturates, or becomes constant, at high $H$. This was usually attributed to extensive ion dissociation and/or detector saturation at high $H[3,30]$. In the evaluation of DI, we included the abundances of all the product ions formed inside and outside the source. That is, the influence of ion dissociation on DI has been already taken care of. We checked whether our measurement was affected by detector saturation. Among the ions appearing in the MALDI spectrum of $\mathrm{Y}_{5} \mathrm{R}$ (Figure 1), $\mathrm{CHCA}$-derived ions such as $\left[\mathrm{CHCA}+\mathrm{H}-\mathrm{H}_{2} \mathrm{O}\right]^{+}$are the most abundant and, hence, might cause the detector saturation. Immonium Y would not be affected because it arrives at the detector earlier than CHCA-derived ions. In contrast, the $\left[\mathrm{Y}_{5} \mathrm{R}+\mathrm{H}\right]^{+}$peak, arriving later than $\mathrm{CHCA}$-derived ions, would be affected if the saturation occurs. As a test, we activated the ion gate to eliminate most of the ions arriving between the immonium $\mathrm{Y}$ and $\left[\mathrm{Y}_{5} \mathrm{R}+\mathrm{H}\right]^{+}$. CHCA-MALDI spectra for $\mathrm{Y}_{5} \mathrm{R}$ recorded without and with ion gate activation are shown in Figure 3. The $\mathrm{Y}:\left[\mathrm{Y}_{5} \mathrm{R}+\mathrm{H}\right]^{+}$ratio measured with the ion gate

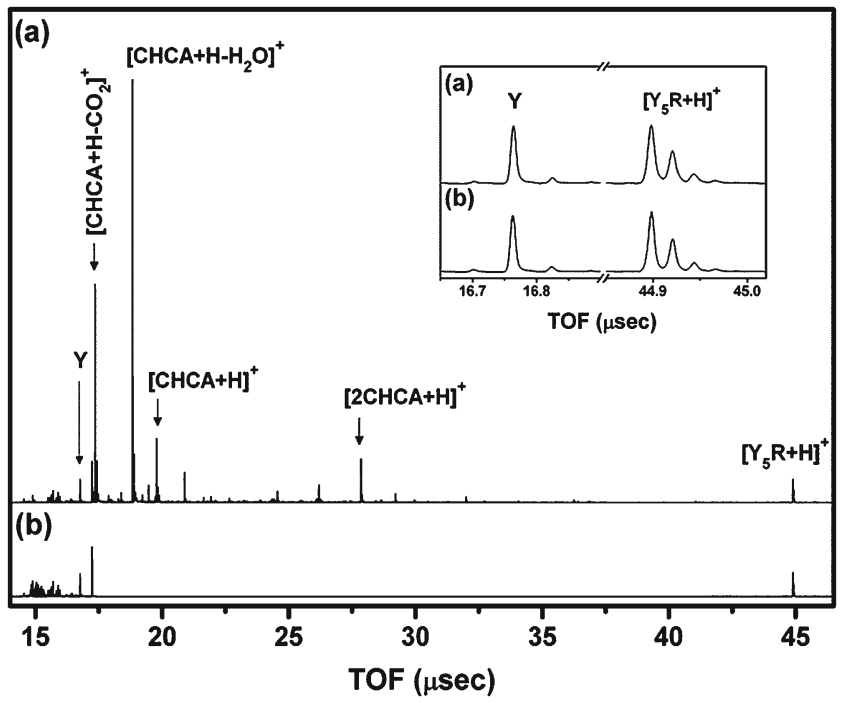

Figure 3. MALDI spectra for $Y_{5} R$ recorded (a) without and (b) with activating the ion gate. Activation of the ion gate in (b) was made to eliminate most of the strong ion signals appearing between the immonium $\mathrm{Y}$ ion and $\left[\mathrm{Y}_{5} \mathrm{R}+\mathrm{H}\right]^{+}$. The $2.0 \mathrm{kV}$ was applied to MCP in both cases activation was $0.61 \pm 0.02$ versus $0.59 \pm 0.03$ measured without, indicating no detector saturation. We also performed other tests (Supplementary Material) and found that the detector saturation was unimportant under our experimental condition. To summarize, neither the extensive ion fragmentation nor the detector saturation is responsible for the observation that DI is unaffected by the laser pulse energy.

In the gas-phase proton transfer (GPPT) hypothesis, primary ions are formed from matrix neutrals (M) via photoexcitation. At the most popular MALDI wavelength of $337 \mathrm{~nm}$, absorption of three photons is needed for the ionization of common matrices. Three-photon ionization is highly unlikely under the usual MALDI condition employing a moderate amount of laser fluence. Hence, various alternative schemes have been proposed. Among these, the exciton pooling scheme is widely thought to be the most promising $[8,31]$. Here, many matrix molecules in the sample are excited by a laser pulse and excitons migrate. Ionization occurs when three or more excitons are pooled in one molecule. Participation of three or more photons suggests a nonlinear nature of the process. In a simulation by Knochenmuss and Zhigilei [32], DI of 2,5-dihydroxybenzoic acid got larger by a factor of five when the laser fluence increased by $30 \%$. Other schemes requiring multiphoton absorption would also display nonlinear response to laser fluence. In this regard, we emphasize our observation that the total number of gas-phase ions (i.e., the sum of peptide- and matrix-derived ions) generated by MALDI was unaffected by the laser pulse energy (Figure 2c). A similar pulse energy independence was observed in MALDI of pure CHCA, even though the result is not shown here because it is virtually the same as Figure 2c. In fact, this is not surprising because the total number of ions from a peptide/ matrix mixture is the same as that from pure CHCA.

One might argue that the fluence independence of DI of pure CHCA simply means that the fluence dependence for the formation of a gas-phase matrix ion is the same as that of a gas-phase neutral and, hence, the two effects cancel in DI. Let us divide the situation into two cases depending on whether the gas-phase matrix ion originates from a matrix cation in the sample (Case I) or from its neutral (Case II). In Case I, the matrix ion originates from a preformed ion and, hence, the laser-induced ionization is not involved. In Case II, laser ionizes the matrix in the solid sample and ablates the ion into the gas phase. Let us suppose that the number of photons needed for the ionization is $\mathrm{n}_{1}$ and those for the ablation of ions and neutrals are $n_{2}$ and $n_{3}$, respectively. Then, accepting the above argument, the fluence independence of DI requires $n_{1}+n_{2}=n_{3}$, or $n_{3}-n_{2}=n_{1} \geq 0$. When $\mathrm{n}_{1}>0, \mathrm{n}_{3}>\mathrm{n}_{2}$ (i.e., more photons are needed for the ablation of a neutral than an ion), which is unlikely; $\mathrm{n}_{1}=0$ is selfcontradictory because the situation becomes photoionization without photoabsorption. Discussion made so far can be summarized as follows. Hypotheses postulating the laserinduced ionization of matrix molecules are incompatible 
with the present experimental result that DI for pure CHCA is unaffected by the laser pulse energy.

At the moment, we do not have an explanation for the general observation that the ion intensity in a single shot spectrum obtained at a laser focal spot of a fresh sample increases rapidly with the laser fluence, especially near the threshold, while the total degree of ionization (i.e., DI) is unaffected. As shown in Supplementary Material, the total ion signal emitted from a laser focal spot at eight times the threshold was larger than those at four times the threshold by around $40 \%$, even though it became the same when integrated over the parallelogram. We also found that the area of the burn mark at the focal spot increased by around $50 \%$ with the above increase in the pulse energy. A small increase in the total ion signal from a focal spot due to larger effective spot size at higher fluence, as suggested by Beavis [21], provides part of the explanation. However, the increase is not large enough to account for rapid increase with the fluence, especially near the threshold, in the ion signal in a single shot spectrum from a fresh sample. Accepting that the ionization efficiency does not increase with the laser fluence, we propose that the increase in ion signal is due to larger material emission at higher fluence. According to the studies on laser ablation of molecular solids by Zhigilei et al. [33], two processes compete near the threshold, thermal desorption and phase explosion (or ablation). Even though the thermal desorption is the main process at low fluence, the ablation suddenly becomes dominant above a certain critical fluence and saturates as schematically shown in Figure 4. We speculate that the so-called threshold fluence in MALDI lies at a point close to the crossover between desorption and ablation and that the sudden increase and saturation of a MALDI signal with the fluence are the characteristics of material emission by ablation.

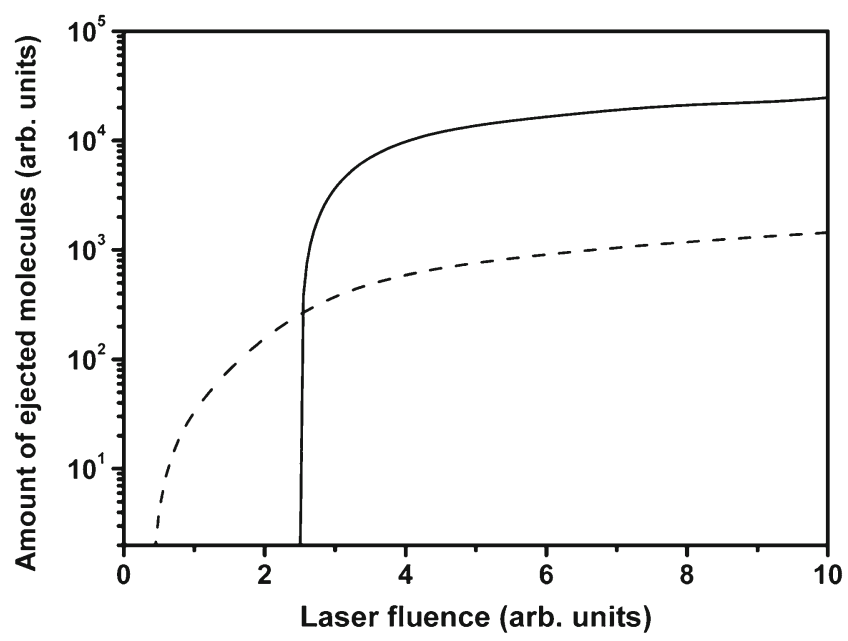

Figure 4. A schematic drawing of the amount of ejected molecules versus laser fluence in laser ablation. The dashed and solid lines denote material ejections by desorption and ablation, respectively

\section{Concentration Dependence of DI}

In our previous CHCA-MALDI study of BTPP-Cl [16], the number of the salt-derived cations increased with the concentration of the salt in the solid sample, whereas that of CHCA-derived ones decreased. However, DIs for both decreased with the salt concentration. Similar suppression effects $[7,8,16,34]$ in peptide MALDI have been known and attributed to the competition for protons by neutral matrix and peptide molecules. To find an explanation for the experimental results for salts, we estimated the equilibrium constant for the neutralization of a cation and an anion, $\mathrm{A}^{+}+$ $\mathrm{B}^{-} \rightarrow \mathrm{A}^{+} \mathrm{B}^{-}$, as $K_{\text {neutral }} \approx \exp (-U / \mathrm{RT})$, where $U$ was the binding energy for the ion pair. Approximating $U$ as the Coulomb potential, $K_{\text {neutral }}$ of $3 \times 10^{14}$ was obtained at $1000 \mathrm{~K}$ when the inter-charge distance was $5 \AA$. This suggested that the release of a preformed ion in a solid into the gas phase would be very difficult. Regardless, the gasphase $\mathrm{BTPP}^{+}$ions were formed, even though weakly, which was explained by two hypotheses. In Hypothesis 1, the gasphase $\mathrm{BTPP}^{+}$originates from the preformed $\mathrm{BTPP}^{+}$in the solid that is dielectrically screened from counter anions by matrix neutrals. Even though tiny in fraction, the existence of such isolated ions may be an intrinsic property of amorphous ionic solids due to entropic disorder. In Hypothesis 2, preformed $\mathrm{BTPP}^{+}$ions in the solid are released as part of neutral ion pairs, which lose the counter anions via gasphase reactions with matrix-derived cations, such as shown in Reaction 1. In Hypothesis 1, the suppression effects may occur because the fractions of the dielectrically screened ionic species in the solid sample will decrease with the analyte concentration. In Hypothesis 2, the number of $\mathrm{BTPP}^{+}$will increase at the cost of matrix-derived ions (matrix suppression) as more ion pairs $\left(\mathrm{BTPP}^{+} \mathrm{Cl}^{-}\right)$are released into the gas phase. However, this increase will slow down at high concentration of ion pairs, eventually resulting in decrease of DI for $\mathrm{BTPP}^{+}$ (analyte suppression).

DI data for the $\mathrm{Y}_{5} \mathrm{R}$-CHCA mixture in Table 1 are drawn in Figure 5. Concentration dependences for the other peptideCHCA mixtures were very similar. Interestingly, they were also very similar to those observed for salts, indicating similar gas-phase ion formation mechanisms for peptides and salts. Rather than jumping to a conclusion, we will try to find plausible pictures by improving the prevailing theories (i.e., the LS and GPPT hypotheses) such that they become compatible with the data obtained in this work.

\section{Modification of the LS Hypothesis}

The key assumptions in the LS hypothesis are $\left(1^{\mathrm{L}}\right)$ peptides are present in the solid sample as a protonated form $\left(\mathrm{AH}^{+}\right.$, preformed), $\left(2^{\mathrm{L}}\right)$ they are desorbed as free $\mathrm{AH}^{+}$or positively charged clusters containing $\mathrm{AH}^{+}$, and $\left(3^{\mathrm{L}}\right)$ some $\mathrm{AH}^{+}$ions are formed from the positively charged clusters. Discarding the cluster ion part, the situation may be explained by Hypothesis 1 ('the dielectrically screened preformed ion 

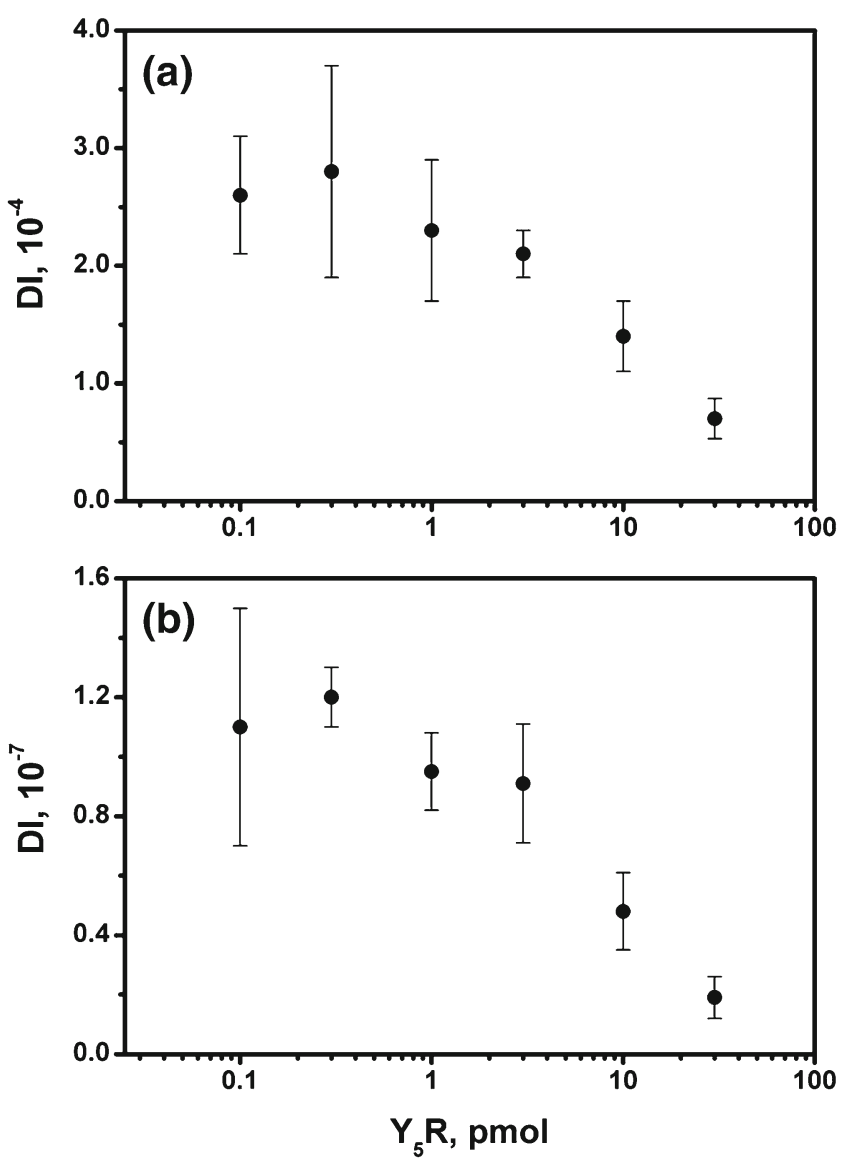

Figure 5. Dls for (a) $Y_{5} R$ and (b) $\mathrm{CHCA}$ versus the amount of $\mathrm{Y}_{5} \mathrm{R}$ in solid samples containing $25 \mathrm{nmol} \mathrm{CHCA}$. The $4.5 \mu \mathrm{J} /$ pulse of laser energy was used

emission') or by Hypothesis 2 ('the ion pair emission/matrixassisted charge separation') derived in the salt study [16]. In both cases, $[\mathrm{CHCA}-\mathrm{H}]^{-}$formed by the following solutionphase reactions will be the counter anion in the ion pair.

$$
\begin{gathered}
\mathrm{CHCA}+\mathrm{CHCA} \leftrightarrow[\mathrm{CHCA}+\mathrm{H}]^{+}+[\mathrm{CHCA}-\mathrm{H}]^{-} \\
\mathrm{Y}_{5} \mathrm{R}+\mathrm{CHCA} \leftrightarrow\left[\mathrm{Y}_{5} \mathrm{R}+\mathrm{H}\right]^{+}+[\mathrm{CHCA}-\mathrm{H}]^{-}
\end{gathered}
$$

\section{Modification of the GPPT Hypothesis}

The key assumptions in Knochenmuss' version of the GPPT hypothesis are $\left(1^{\mathrm{G}}\right)$ peptides are desorbed as neutrals, $\left(2^{\mathrm{G}}\right)$ primary ions are generated by laser-induced ionization of matrix molecules, and $\left(3^{\mathrm{G}}\right)$ proton transfer from a matrixderived ion to a peptide neutral generates a peptide ion. Discarding the assumption $\left(2^{\mathrm{G}}\right)$, the hypothesis becomes as follows. 'Laser ablation of a solid sample releases neutral peptides and matrix-derived materials. Proton transfer from a matrix-derived material to a neutral peptide generates a gasphase peptide ion.'
Both the neutral matrix molecule and $\mathrm{M}^{\prime} \mathrm{H}^{+}$can be the proton donor in the gas-phase proton transfer, as illustrated in Reactions 3 and 4.

$$
\mathrm{Y}_{5} \mathrm{R}+\mathrm{M}^{\prime} \mathrm{H}^{+} \leftrightarrow\left[\mathrm{Y}_{5} \mathrm{R}+\mathrm{H}\right]^{+}+\mathrm{M}^{\prime}
$$

According to the data in Table 1 and in the tables in Supplementary Material, only $10^{-7}$ of CHCA molecules are in one of the protonated forms. However, since $\mathrm{M}^{\prime} \mathrm{H}^{+}$is a stronger acid than CHCA, Reaction 4 will have the larger equilibrium constant than Reaction 3 and, hence, will effectively compete with the latter reaction. Furthermore, the suppression effect will not be observed when the neutral matrix molecules are the proton donors because they are far more abundant than the analyte, suggesting $\mathrm{M}^{\prime} \mathrm{H}^{+}$as the proton donor. Then, the GPPT hypothesis is further refined as follows. 'Materials emitted in the ablation of a peptidematrix mixture include neutral peptides and matrix-derived acidic cations $\left(\mathrm{M}^{\prime} \mathrm{H}^{+}\right)$. Gas-phase proton transfer from $\mathrm{M}^{\prime} \mathrm{H}^{+}$ to a peptide generates a protonated peptide.' We will call this Hypothesis 3. Unlike in the original GPPT hypothesis, however, $\mathrm{M}^{\prime} \mathrm{H}^{+}$is not formed via laser-induced ionization, but presumably via thermal reactions such as auto-ionization (Reaction 2) in condensed phases.

\section{Thermal Explanation for MALDI}

So far, we have modified two prevailing hypotheses for peptide MALDI to accommodate the degree of ionization data we obtained. Two hypotheses emerged from the LS hypothesis, (i.e. 'the dielectrically screened preformed ion emission (Hypothesis 1)' and 'the ion pair emission/matrix-assisted charge separation (Hypothesis 2)'. Hypothesis 3 that is an improved version of the GPPT hypothesis may retain its name. However, it is drastically different from the original version in the sense that primary ions are not formed by the laser-induced ionization of matrix molecules anymore.

The fact that the sum of the peptide- and matrix-derived ions was the same regardless of the peptide concentration might be taken as evidence for Hypothesis 3. However, this is also compatible with Hypotheses 1 and 2, as found for salts. At the moment, we do not know which of the three hypotheses are dominant. Regardless, one thing is clear (i.e. the driving force for the gas-phase ion generation in MALDI is the thermal energy gained by the sample through the electronic excitation of matrix molecules followed by intramolecular relaxation processes such as the internal conversion.

Many years ago, Vertes et al. [35] developed a hydrodynamic model for MALDI, which provided a general mathematical framework to probe the fate of the deposited energy in terms of solid- and gas-phase processes. Calculations utilizing information available at that time resulted in the surface temperature of $500 \mathrm{~K}$ for nicotinic acid at $10 \mathrm{~ns}$ after laser pulse. Then, the temperature of the gas-phase matrix dropped to $110 \mathrm{~K}$ due to expansion cooling. Other 
results indicated higher temperatures in MALDI [20, 25, 3639]. For example, our own determination of the effective temperature by a kinetic method resulted in around 900 and $450 \mathrm{~K}$ for the 'early' and 'late' CHCA plumes, respectively $[20,25,39]$. That is, the hydrodynamic model correctly predicted the occurrence of expansion cooling in the matrix plume, even though the results were not accurate enough. Since more data have become available after the formulation of the model, it will be interesting to see if hydrodynamic calculations utilizing new information can provide better insights on the physical and chemical processes occurring in MALDI.

\section{Conclusion}

Degrees of ionization in peptide MALDI were measured and used to evaluate and improve two prevailing hypotheses for gas-phase peptide ion formation in MALDI (i.e., the LS and GPPT hypotheses). Specifically, the cluster ion part of the LS hypothesis and the primary ion formation by laserinduced ionization of matrix molecules in the GPPT hypothesis were discarded based on the experimental data. Instead, a very simple thermal picture for the gas-phase ion formation was proposed, as follows. MALDI releases a peptide into the gas phase either as a free protonated peptide (Hypothesis 1), as a protonated peptide in a neutral ion pair (Hypothesis 2), or as a neutral peptide (Hypothesis 3). In Hypotheses 2 and 3, peptide ions were assumed to form via gas-phase reactions of the neutrals with matrix-derived cations. To accommodate low DI for peptides, the ion emission in Hypothesis 1 was assumed to occur only for dielectrically screened preformed ions in a solid sample. With the laser-induced ionization discarded, gas-phase CHCA-derived ions would also originate from the preformed ions in the solid sample. The main strength of the above hypotheses is that they are simple but, at the same time, are compatible with the experimental data.

\section{Acknowledgments}

The authors acknowledge support for this work by the National Research Foundation of Korea (NRF) grant funded by the Korea government (MEST) (305-20110059). Y.J.B. and Y.S.S. thank the Ministry of Education, Science, and Technology, Republic of Korea, for Brain Korea 21 Fellowship.

\section{References}

1. Hillenkamp, F., Karas, M.: The MALDI process and method. In: Hellenkamp, F., Peter-Katalinić, J. (eds.) MALDI MS. A practical guide to instrumentation, methods and applications, p. 1. Wiley-VCH, Weinheim (2007)

2. Cole, R.B.: Electrospray and MALDI mass spectrometry. Fundamentals, instrumentation, practicalities, and biological applications, 2nd ed. Wiley: Hoboken (2010)

3. Dreisewerd, K.: The desorption process in MALDI. Chem. Rev. 103, 395-425 (2003)
4. Zenobi, R., Knochenmuss, R.: Ion formation in MALDI mass spectrometry. Mass Spectrom. Rev. 17, 337-366 (1998)

5. Knochenmuss, R., Stortelder, A., Breuker, K., Zenobi, R.: Secondary ion-molecule reactions in matrix-assisted laser desorption/ionization. $J$. Mass Spectrom. 35, 1237-1245 (2000)

6. Breuker, K., Knochenmuss, R., Zhang, J., Storteler, A., Zenobi, R.: Thermodynamic control of final ion distributions in MALDI: in-plume proton transfer reactions. Int. J. Mass Spectrom. 226, 211-222 (2003)

7. Knochenmuss, R., Zenobi, R.: MALDI Ionization: The role of in-plume processes. Chem. Rev. 103, 441-452 (2003)

8. Knochenmuss, R.: Ion formation mechanisms in UV-MALDI. Analyst 131, 966-986 (2006)

9. Karas, M., Glückmann, M., Schäfer, J.: Ionization in matrix-assisted laser desorption/ionization: singly charged molecular ions are the lucky survivors. J. Mass Spectrom. 35, 1-12 (2000)

10. Karas, M., Krüger, R.: Ion formation in MALDI: The cluster ionization mechanism. Chem. Rev. 103, 427-439 (2003)

11. Jaskolla, T.W., Karas, M.: Compelling evidence for lucky survivor and gas phase protonation: The unified MALDI analyte protonation mechanism. J. Am. Soc. Mass Spectrom. 22(6), 976-988 (2011)

12. Ens, W., Mao, Y., Mayer, F., Standing, K.G.: Properties of matrixassisted laser desorption. Measurements with a time-to-digital converter. Rapid Commun. Mass Spectrom. 5(3), 117-123 (1991)

13. Puretzky, A.A., Geohegan, D.B.: Gas-phase diagnostics and LIFimaging of 3-hydroxypicolinic acid MALDI-matrix plumes. Chem. Phys. Lett. 286, 425-432 (1998)

14. Mowry, C.D., Johnston, M.V.: Simultaneous detection of ions and neutrals produced by matrix-assisted laser desorption. Rapid Commun. Mass Spectrom. 7, 569-575 (1993)

15. Quist, A.P., Huth-Fehre, T., Sundqvist, B.U.R.: Total yield measurements in matrix-assisted laser desorption using a quartz crystal microbalance. Rapid Commun. Mass Spectrom. 8, 149-154 (1994)

16. Moon, J.H., Shin, Y.S., Bae, Y.J., Kim, M.S.: Ion yields for some salts in MALDI: mechanism for the gas-phase ion formation from preformed ions. J. Am. Soc. Mass Spectrom. 23, 162-170 (2012)

17. Yoon, S.H., Kim, M.S.: Development of a time-resolved method for photodissociation mechanistic study of protonated peptides: Use of a voltage-floated cell in a tandem time-of-flight mass spectrometer. J. Am. Soc. Mass Spectrom. 18, 1729-1739 (2007)

18. Bae, Y.J., Yoon, S.H., Moon, J.H., Kim, M.S.: Optimization of reflectron for kinetic and mechanistic studies with multiplexed multiple tandem $\left(\mathrm{MS}^{\mathrm{n}}\right)$ time-of-flight mass spectrometry. Bull. Korean Chem. Soc. 31(1) 92-99 (2010)

19. Yoon, S.H., Moon, J.H., Choi, K.M., Kim, M.S.: A deflection system to reduce the interference from post-source decay product ions in photodissociation tandem time-of-flight mass spectrometry. Rapid Commun. Mass Spectrom. 20, 2201-2208 (2006)

20. Yoon, S.H., Moon, J.H., Kim, M.S.: Changes in dissociation efficiency and kinetics of peptide ions induced by basic residues and their mechanistic implication. J. Am. Soc. Mass Spectrom. 22, 214-220 (2011)

21. Beavis, R. C.: Phenomenological models for matrix-assisted laser desorption ion yields near the threshold fluence. Org. Mass Spectrom. 27, 864-868 (1992)

22. Moon, J.H., Yoon, S.H., Kim, M.S.: Temperature of peptide ions generated by matrix-assisted laser desorption ionization and their kinetic parameters. J. Phys. Chem. B 113, 2071-2076 (2009)

23. Beavis, R.C., Chait, B.T.: Factors affecting the ultraviolet laser desorption of proteins. Rapid Commun. Mass Spectrom. 3(7), 233237 (1989)

24. Westman, A., Brinkmalm, G. Barofsky, D.F.: MALDI induced saturation effects in chevron microchannel plate detectors. Int. J. Mass Spectrom. Ion Processes 169/170, 79-87 (1997)

25. Yoon, S.H., Moon, J.H., Kim, M.S.: A comparative study of in- and post-source decays of peptide and preformed ions in matrix-assisted laser desorption ionization time-of-flight mass spectrometry: Effective temperature and matrix effect. J. Am. Soc. Mass Spectrom. 21, 18761883 (2010)

26. Nishikaze, T., Takayama, M.: Disappearance of interfering alkali-metal adducted peaks from matrix-assisted laser desorption/ionization mass spectra of peptides with serine addition to $\alpha$-cyano-4-hydroxycinnamic acid matrix. Rapid Commun. Mass Spectrom. 21, 3345-3351 (2007)

27. Dashtiev, M., Wäfler, E., Röhling, U., Gorshkov, M., Hillenkamp, F., Zenobi, R.: Positive and negative analyte ion yield in matrix-assisted laser desorption/ionization. Int. J. Mass Spectrom. 268, 122-130 (2007) 
28. Musapelo, T., Murray, K.K.: Particle formation in ambient MALDI plumes. Anal. Chem. 83, 6601-6608 (2011)

29. Dreisewerd, K., Schürenberg, M., Karas, M., Hillenkamp, F.: Influence of the laser intensity and spot size on the desorption of molecules and ions in matrix-assisted laser desorption/ionization with a uniform beam profile. Int. J. Mass Spectrom. Ion Processes 141, 127-148 (1995)

30. Westmacott, G., Ens, W., Hillenkamp, F., Dreisewerd, K., Schürenberg, M.: The influence of laser fluence on ion yield in matrix-assisted laser desorption ionization mass spectrometry. Int. J. Mass Spectrom. 221, 67-81 (2002)

31. Knochenmuss, R.: A quantitative model of ultraviolet matrix-assisted laser desorption/ionization. J. Mass Spectrom. 37, 867-877 (2002)

32. Knochenmuss, R., Zhigilei, L.V.: Molecular dynamics model of ultraviolet matrix-assisted laser desorption/ionization including ionization processes. J. Phys. Chem. B 109, 22947-22957 (2005)

33. Zhigilei, L.V., Leveugle, E., Garrison, B.J., Yingling, Y.G., Zeifman, M.I.: Computer simulations of laser ablation of molecular substrates. Chem. Rev. 103, 321-327 (2003)
34. Knochenmuss, R., Dubois, F., Dale, M.J., Zenobi, R.: The matrix suppression effect and ionization mechanisms in matrix-assisted laser desorption/ionization. Rapid Commun. Mass Spectrom. 10, 871-877 (1996)

35. Vertes, A., Irinyi, G., Gijbels, R.: Hydrodynamic model of matrixassisted laser desorption mass spectrometry. Anal. Chem. 65, 23892393 (1993)

36. Mowry, C.D., Johnston, M.V.: Internal energy of neutral molecules ejected by matrix-assisted laser desorption. J. Phys. Chem. 98, 19041909 (1994)

37. Campbell, J.M., Vestal, M.L., Blank, P.S., Stein, S.E., Epstein, J.A., Yergey, A.L.: Fragmentation of leucine enkephalin as a function of laser fluence in a MALDI TOF-TOF. J. Am. Soc. Mass Spectrom. 18, 607-616 (2007)

38. Koubenakis, A., Frankevich, V., Zhang, J., Zenobi, R.: Time-resolved surface temperature measurement of MALDI matrices under pulsed UV laser irradiation. J. Phys. Chem. A 108, 2405-2410 (2004)

39. Bae, Y.J., Moon, J.H., Kim, M.S.: Expansion cooling in the matrix plume is under-recognized in MALDI mass spectrometry. J. Am. Soc. Mass Spectrom. 22, 1070-1078 (2011) 